\title{
Extensive Performance Studies for the ATLAS BIS-MDT Precision Muon Chambers With Cosmic Rays
}

T. Alexopoulos, R. Avramidou, U. Bratzler, C. Cernoch, M. Dris, D. Fassouliotis, T. A. Filippas, E. Fokitis, E. N. Gazis, P. Ioannou, E. C. Katsoufis, Ch. Kourkoumelis, A. Krepouri, T. Liolios, S. Maltezos, M. Manolopoulou, S. Palestini, Ch. Petridou, V. Polychronakos, D. Sampsonidis, P. Savva, G. Stavropoulos, G. Tsipolitis, E. Tzamariudaki, Ch. Valderanis, J. Wotschack, and S. Zimmermann

\begin{abstract}
ATLAS (a toroidal LHC apparatus) is a general purpose experiment that will start its operation at the large hadron collider (LHC) at CERN in 2007. The ATLAS detector is designed to explore numerous physics processes by recording, measuring, and investigating the products emerging from proton-proton collisions at energies up to $14 \mathrm{TeV}$. High-precision muon momentum measurement $\left(\mathrm{dp} / \mathrm{p} \sim 10 \%\right.$ at $\left.\mathrm{p}_{\mathrm{T}}=1 \mathrm{TeV} / \mathrm{c}\right)$ over large areas using monitored drift tube (MDT) chambers is crucial for the ATLAS experiment. More than 1200 MDT chambers, consisting of approximately 370000 drift tubes, will provide a total coverage of $5500 \mathrm{~m}^{2}$. Three Greek universities have taken the responsibility to construct 130 barrel inner small (BIS)-MDT chambers using 30000 drift tubes of $\sim 1.7 \mathrm{~m}$ length that have been quality tested before assembly. The design of the muon drift tubes aims at high detection efficiency $(>95 \%)$ and a spatial single tube resolution of $<80 \mu \mathrm{m}$. This paper describes the cosmic ray test setup, which has been instrumented in order to verify that the BIS-MDT chamber Module- 0 fulfills its design requirements. The analysis of its data shows that the chamber meets these requirements; it has low noise levels, uniform drift properties, good spatial resolution, and high particle detection efficiency.
\end{abstract}

Index Terms-ATLAS, cosmic rays, gas detectors, muon spectrometer, particle detectors.

\section{INTRODUCTION}

$\mathbf{T}$ HE ATLAS detector for the large hadron collider (LHC) at CERN [1], the construction of which has already started, will study the products of proton-proton collisions at c.m.s.

Manuscript received February 24, 2003; revised May 29, 2003.

T. Alexopoulos, R. Avramidou, U. Bratzler, M. Dris, T. A. Filippas, E. Fokitis, E. N. Gazis, E. C. Katsoufis, S. Maltezos, P. Savva, G. Tsipolitis, and E. Tzamariudaki are with the Physics Department, National Technical University of Athens, Zografou, GR-15780 Athens, Greece (e-mail: egazis@central.ntua.gr).

C. Cernoch, S. Palestini, and J. Wotschack are with CERN, CH-1211 Geneva, Switzerland.

D. Fassouliotis, P. Ioannou, and C. Kourkoumelis are with the Physics Department, University of Athens, Panepistimioupoli Zografou, GR-15771 Athens, Greece.

A. Krepouri, T. Liolios, M. Manolopoulou, C. Petridou, D. Sampsonidis, and C. Valderanis are with the Physics Department, University of Thessaloniki, GR-54006 Thessaloniki, Greece.

V. Polychronakos is with the Physics Department, Brookhaven National Laboratory, Upton, NY 11973-5000 USA.

G. Stavropoulos was with the Physics Department, National Technical University of Athens, Zografou, GR-15780 Athens, Greece. He is now with the Physics Division, Lawrence Berkeley National Laboratory, Berkeley, CA 94720 USA.

S. Zimmermann is with the Faculty of Physics, University of Freiburg, 79104

Freiburg, Germany.

Digital Object Identifier 10.1109/TNS.2003.820630

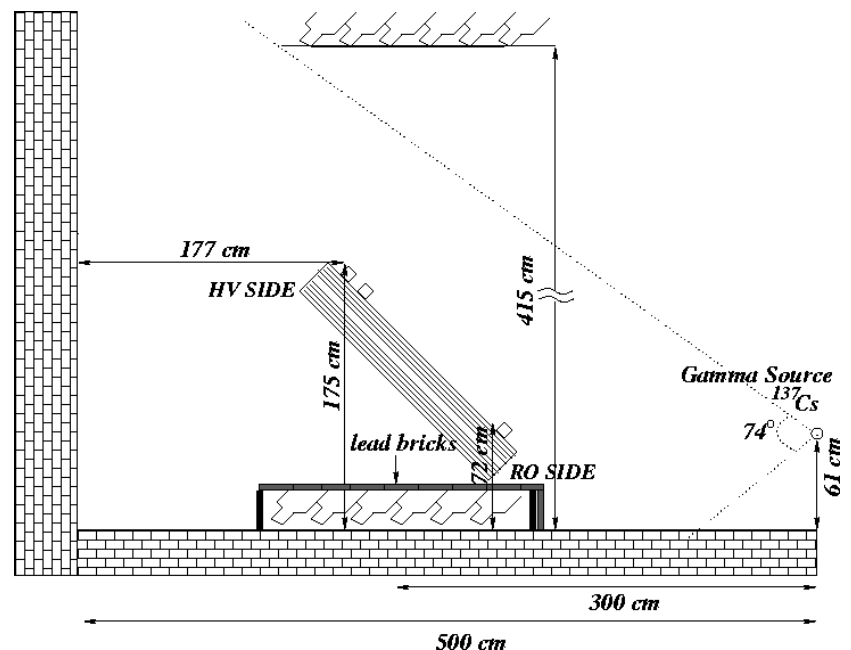

Fig. 1. Hodoscope and the BIS-MDT chamber setup at the X5/GIF area. The chamber is inclined by $\sim 45^{\circ}$ to detect both the cosmic ray muons and the source photons.

energies of up to $14 \mathrm{TeV}$. The outermost part of the ATLAS detector, the muon spectrometer, is designed to identify muons with high efficiency, and measure their momenta with a resolution of $\sim 10 \%$ at $\mathrm{p}_{\mathrm{T}}=1 \mathrm{TeV} / \mathrm{c}$. The measurement is performed inside the toroidal field created by powerful air-core toroid magnets with the help of the ATLAS muon precision tracking system.

A cosmic ray setup has been constructed (Fig. 1), in order to verify that the BIS-MDT chamber Module-0 fulfills the specifications of the ATLAS muon spectrometer. The measurements took place in the Gamma Irradiation Facility (GIF) [2] at CERN, which is a test area, where muon detectors are exposed to a high-energy muon beam in the presence of an adjustable high background flux of photons, with the purpose of simulating the operating environment of the LHC. Our results with cosmic rays have been used as a reference for the later study of the muon chamber performance and for the aging tests at the CERN $\mathrm{X} 5 / \mathrm{GIF}$ area.

The setup consists of a hodoscope [3], which serves as a trigger system and the BIS-MDT chamber [4]. The hodoscope $\left(1.05 \times 1.07 \mathrm{~m}^{2}\right)$ covers almost completely the muon chamber and it provides the transverse and longitudinal coordinate of the incident muon for the pattern recognition, for the track reconstruction and for the correction of the signal propagation 
along the wire. The BIS-MDT chamber, constructed in Greece, consists of two multilayers composed of four layers each. Each layer consists of 30 aluminum drift tubes $1700 \mathrm{~mm}$ long (including the endplugs). The multilayers are separated by seven $6 \mathrm{~mm}$ thick aluminum strips [5], [6]. Each individual drift tube has undergone a series of quality controls before its gluing on the chamber [7], [8]. The BIS-MDT chamber construction quality has been checked at the CERN X-ray tomograph facility in order to verify that it meets the specification requirements [9].

The cosmic muon rate is $\sim 7 \mathrm{~Hz}$, in agreement with calculations, taking into account that the overall angular distribution of muons at ground level is proportional to $\cos ^{2} \theta$ (characteristic of muons with energy $\sim 3 \mathrm{GeV}$ ) and that the flux per unit solid angle per unit horizontal area about the vertical direction is $\mathrm{I}_{\nu}=80 \mathrm{~m}^{-2} \mathrm{~s}^{-1} \mathrm{sr}^{-1}$ [10]. A small difference between measurements and calculations, of the order of $5 \%$, is due to the inefficiency of the system (gaps between the scintillators).

Below the scintillator upper layer there is an iron layer of $1 \mathrm{~cm}$ width and an additional piece of lead of $2 \mathrm{~cm}$, which covers $3 / 4$ of the scintillator overlap area. The lower scintillators layer is covered by lead bricks of $5 \mathrm{~cm}$ thickness plus an iron layer of $1 \mathrm{~cm}$ width. The stopping power of the existing material puts an energy cut of $\sim 0.2 \mathrm{GeV}$.

The mean energy of the muons at the ground is $\sim 4 \mathrm{GeV}$ [10]. Their most probable energy is $\sim 1 \mathrm{GeV}$, as it comes from Monte Carlo simulation [11].

\section{Performance of the BIS-MDT Chamber}

\section{A. Introduction}

The basic detection element of the MDT chambers is a precision aluminum drift tube of $30 \mathrm{~mm}$ diameter and $400 \mu \mathrm{m}$ wall thickness, with a $50 \mu \mathrm{m}$ diameter gold-plated W-Re wire [12], [13]. The single-wire resolution is typically $80 \mu \mathrm{m}$. To improve the resolution and the efficiency of a chamber beyond the single-wire limit, the BIS-MDT chambers consist of two multilayers of 4 layers each that are glued on either side of a support structure.

The BIS-MDT chamber (Fig. 2) is fully equipped with a gas system and electronics [14], [15]. There is a parallel gas supply for each tube and multiplayer, and the gas flow is $35 \mathrm{Nl} / \mathrm{h}$ (1 volume change per day). The gas is the nominal MDT gas of $\mathrm{Ar}(93 \%) / \mathrm{CO}_{2}(7 \%)$ at a pressure of 3 bar (absolute). The applied high voltage is $3080 \mathrm{~V}$, which corresponds to a gas gain $2 \times 10^{4}$.

Each MDT is read out by an amplifier-shaper-discriminator circuit at one end and the other end is terminated in the characteristic impedance of the tube $(380 \Omega)$. The preamplifier input impedance is relatively low $\sim 100 \Omega$ in order to maximize the collected charge. The shaper has a peaking time of $15 \mathrm{~ns}$. The nominal discriminator threshold is the $60 \mathrm{mV}$ that corresponds to the arrival of the 20th primary electron or $6 \sigma_{\text {noise }}$ [14]. The time measurement is taken by a TDC of 32 bits with time resolution of $250 \mathrm{ps}(\mathrm{rms})$ and bin size of $0.78 \mathrm{~ns}$. The data of each TDC are read out individually via a serial link to a single chamber service module (CSM), which puts them in registers and is responsible for the multiplexing. A special VME version

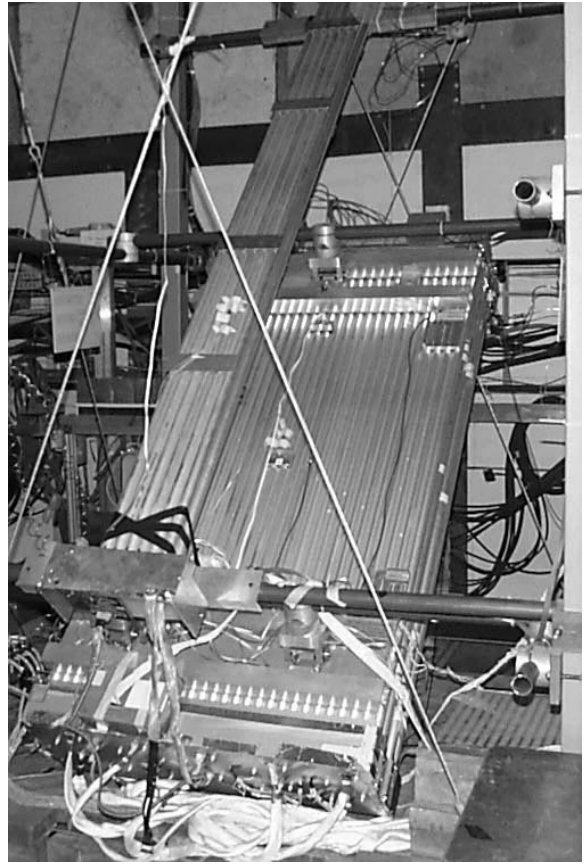

Fig. 2. The BIS-MDT chamber Module-0 at the X5/GIF area at CERN.

of CSM-0 is used, which also does the event building and provides an internal $40 \mathrm{MHz}$ clock for the simulation of the LHC proton-proton collisions.

The data acquisition system is based on a $\mathrm{C}$ language program written in a LabWindows/CVI environment. There is also a slow control data program in LabVIEW for the registration of parameters like pressure, gas flow, high voltage, high-voltage current, and temperature.

About 13000000 cosmic ray events have been collected in order to have high statistics. Some of them have been taken using different high voltage and different discriminator threshold values for the study of the dependence of the drift properties on these variations.

The analysis [16] includes typical drift time spectra, autocalibration studies, track reconstruction derivation, spatial resolution and efficiency studies. Simulation studies using the GARFIELD program [17] on muon drift tube parameters have also been done.

\section{B. Individual Tube Time Spectrum}

The drift time spectrum (Fig. 3) is obtained for each drift tube coming out from all the detected muons, taking into account only the first hit of each event. ${ }^{1}$ The values of the characteristic fit parameters are obtained by fitting the time spectrum with an appropriate function. The nonexistence of extra hits on the right and left sides of the spectrum is an indication of low noise. ${ }^{2}$ The nonlinearity of the $\mathrm{r}-\mathrm{t}$ relation for the $\mathrm{Ar}-\mathrm{CO}_{2}$ mixture becomes evident from the time distribution and it is confirmed by comparison with the simulated drift velocity curve (Fig. 4).

\footnotetext{
${ }^{1}$ The gas, which has been selected for aging reasons, has the disadvantage of its relatively slow and not linear drift properties. The long drift time and the nonlinearity in conjunction with the bipolar shaping cause multiple threshold crossings, from which we keep only the first one.

${ }^{2}$ This concerns mainly the noise due to bad grounding, bad connections, pickup noise etc.
} 


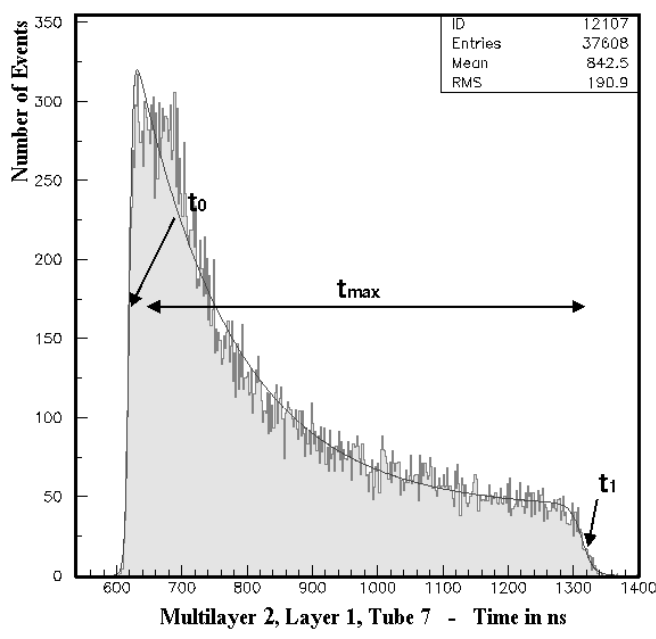

Fig. 3. Typical time distribution of the muon hits for a single tube

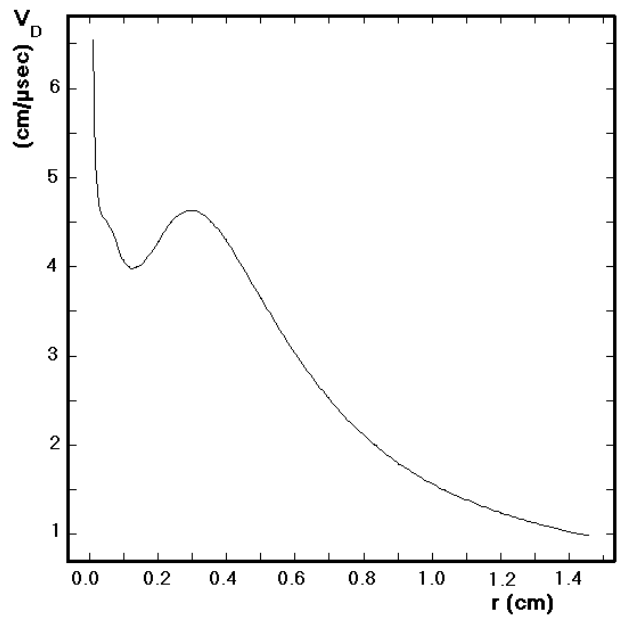

Fig. 4. Electron drift velocity as a function of the distance from the wire (Garfield simulation program).

The double peak in the time distribution is explained by the corresponding double peak of the electron drift velocity, as the event number $\mathrm{dN}$ at a time $\mathrm{dt}$ is proportional to the drift velocity $\mathrm{u}(\mathrm{dN} / \mathrm{dt}=\mathrm{dN} / \mathrm{dx} \cdot \mathrm{dx} / \mathrm{dt}=\mathrm{c} \cdot \mathrm{u})$.

The physical time window ${ }^{4}$, which corresponds to hits induced by muons, is characterized by the start point $t_{0}$ (half of the rise time-leading edge), the end point $t_{1}$ (half of the falling time-trailing edge) and the width defined as $t_{\max }=t_{1}-t_{0}$. The value of $t_{0}{ }^{5}$, which is determined from the fit does not depend

${ }^{3}$ In this distribution, 230 out of 240 drift tube maximum times were plotted. This was because almost all of the tubes of the same mezzanine electronic card (24 drift tubes) appeared as distorted TDC spectra (different shape, more hits, additional peak at the trailing edge, larger $t_{\max }$ ). As this was explained as an electronic effect (cross talk problem), the worst cases were not taken into account.

${ }^{4}$ The physical time window is defined by the point $t_{0}$ (half of the rise timeleading edge), which is related to the tube resolution and affected by the diffusion and clustering effects and the end point $t_{1}$ (half of the falling time-trailing edge). As there are hits coming earlier than $\mathrm{t}_{0}$, which are also induced by cosmic muons, we define the actual time window as the time window-between the first and the last hit.

${ }^{5}$ The error on the determination of $t_{0}$ is of the order of $400 \mathrm{ps}$ for number of events larger or equal to $10^{4}$ and in this case does not contribute to the $\mathrm{r}$ - $\mathrm{t}$ relation errors.

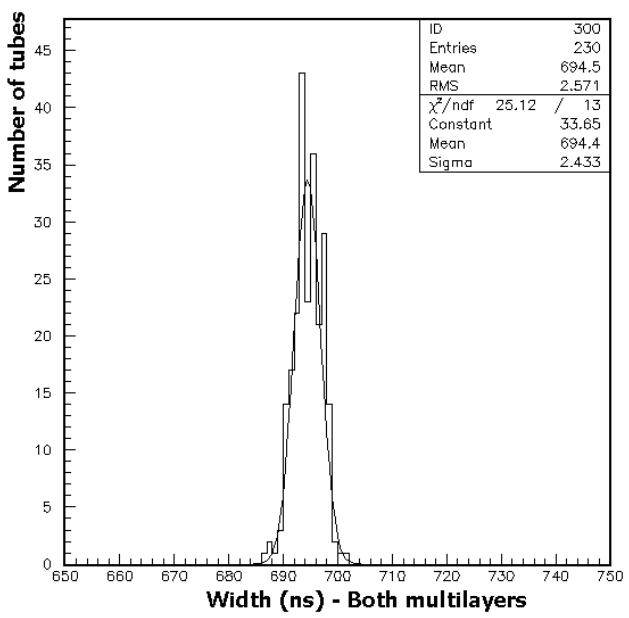

Fig. 5. Distribution ${ }^{3}$ of the maximum drift time $t_{\max }$ for the tubes of the BIS-MDT Module-0 chamber.

on the drift characteristics of the tube and so it does not provide any relevant information. Its only function is to equalize the starting point time distributions of the tubes, which are used for the computation of the space-time relation.

Contrary, the value of $t_{\max }$ depends on the drift properties of the tubes and for this reason is an indicator of the uniform operational behavior of the chamber. Fig. 5 shows the distribution of $t_{\max }$ for the tubes of the BIS-MDT chamber. Its root mean square (rms) amounts to $\sim 2 \mathrm{~ns}$ and it is comparable with the statistical error in the determination of $t_{\max }$, which is dominated by the error on $t_{1}$ due to low statistics. This means that the same space-time relation, which depends on temperature, pressure, gas mixture, and magnetic field can be applied for all the tubes.

\section{High Voltage and Threshold Scans}

The drift properties of the chamber are functions of the electric field. So measurements have been taken at different HV values for a better understanding of these variations. In Fig. 6(a) the TDC spectra for three different HV values are presented, whereas in Fig. 6(b) the left side of the spectrum has been enlarged.

The rise time changes with $\mathrm{HV}$, as the gas amplification increases with the applied voltage. The rise time changes with the increase of the high voltage because the drift velocity increases due to the stronger electric field. This remark could also be explained by the fact that a smaller number of primary electrons (due to increase of gas amplification) is required for the threshold crossing (for a fixed peaking time of the electronics).

There is a similar result for the quantity $t_{0}[$ Fig. 7(b)]. The effect of the different $\mathrm{HV}$ values is more evident for the quantity $t_{\max }$, which decreases with increasing electric field $\left(t_{\max } \propto\right.$ $\left.\left(\nu_{\text {drift }}\right)^{-1} \propto(\mathrm{p} / \mathrm{E})\right)$.

The variation of the discriminator threshold provides additional information to the HV scan. The increase of the threshold discriminator changes the time $t_{0}$, which is visible in the plot (Fig. 7), because a larger number of primary electrons is required for the threshold crossing. The quantity $t_{\max }$ is affected by larger measurement errors, due to the relatively poor statistics in the determination of the $t_{1}$ quantity, which obscure its 


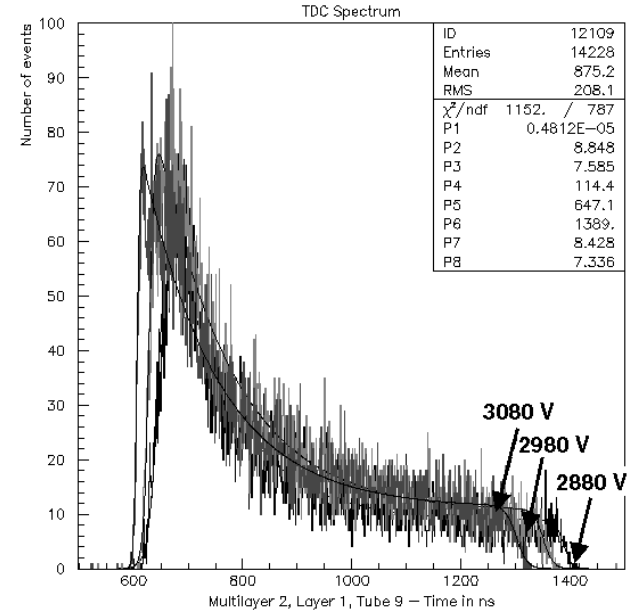

(a)

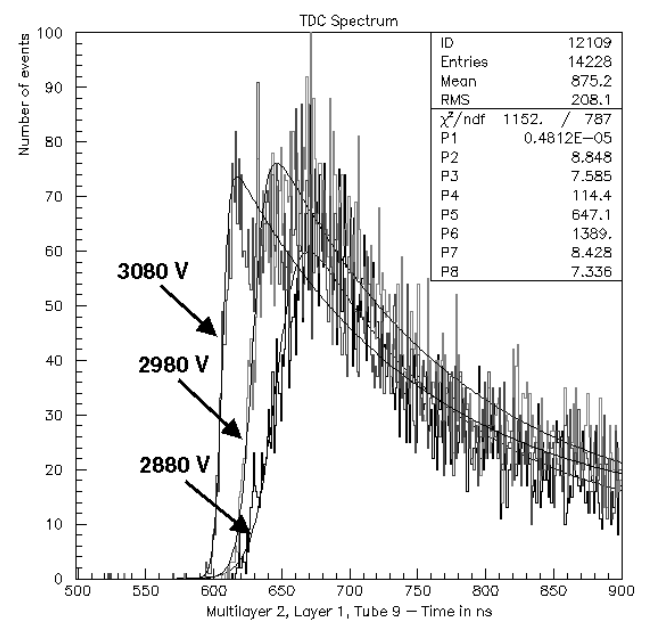

(b)

Fig. 6. (a) TDC spectra for three different HV values of the MDT operation (b) TDC spectra for three different HV values, which are focused on their leading edges.

dependence on the threshold and are expected to be similar to $t_{0}$ for the same reasons.

\section{Autocalibration Method}

One of the most important steps to reconstruct tracks is the computation of the space-time relation [18]-[20] (Fig. 8). It has been performed using the CALIB program [16], which is based on the following method [20]. The TDC spectra are derived for each MDT, and the $t_{0}$ fit parameter, which corresponds to the minimum drift time, is obtained for each tube. This information is used for the determination of the r-t relation.

The procedure is the following. A first approximation r-t relation is used, tracks are reconstructed in the full data sample and the residuals, between each drift circle attributed to the track and the distance of minimum approach of the fitted track to the tube center, as a function of the measured drift time, are calculated. The residuals are taken separately for negative and positive values, which correspond to the two directions of drift in the tube. The values of the $r-t$ relation corrections are extracted from the histograms by taking the mean of a Gaussian fitted to the residual data in each drift time slice. The corrected r-t re-
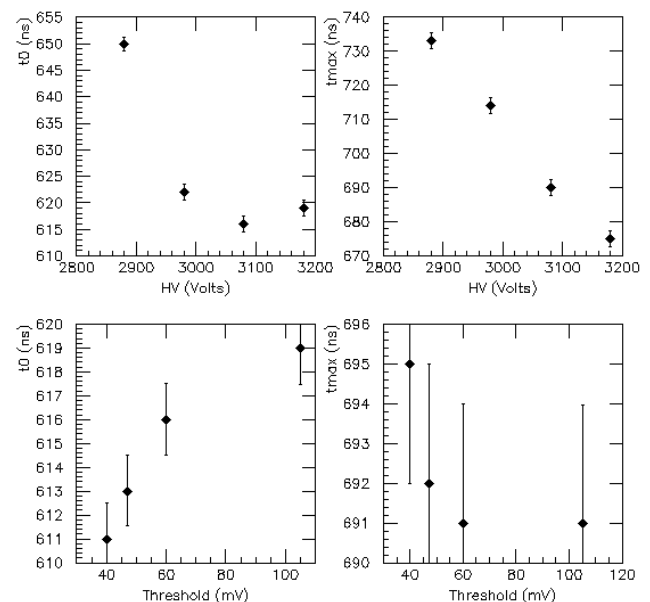

Fig. 7. Dependence of parameters $t_{\max }$ and $t_{0}$ on the MDT HV values and the discriminator threshold.

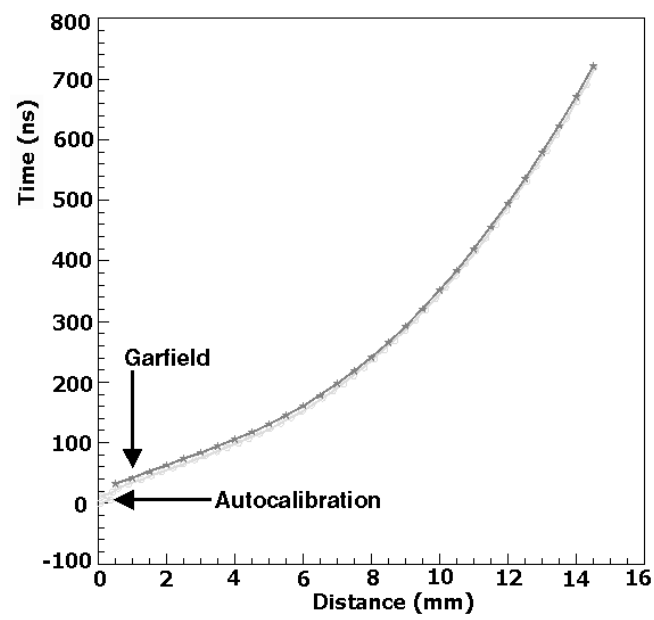

Fig. 8. Space-time relations coming out from the Garfield simulation program and the autocalibration procedure.

lation is used for the next iteration. This task is repeated until a maximum number of iterations is reached, converging to the final $\mathrm{r}$-t relation. In this part of the analysis 10 iterations have been used (the r-t relation is equal within errors to the previous one). This number depends on the goodness of the initial r-t relation. The initial $r-t$ relation that has been used was coming out from the analysis of similar chambers in high-energy muon beam (CERN H8 test beam [21]).

For the autocalibration procedure it is necessary for the muons to have an angular spread to avoid errors coming from track mirror symmetry. The required angular spread that provides a systematic error of $10 \mu \mathrm{m}$ in the measurement of the r-t relation is $\sim 100 \mathrm{mrad}$ [19]. In our case, this requirement is fulfilled as the cosmic muons impinge in different angles, which range, depending on the setup geometry, between $0^{\circ}$ and $14^{\circ}(0-244 \mathrm{mrad})$.

\section{E. Spatial Resolution}

The single tube resolution is obtained by selecting tracks with eight consecutive hits. One of the hits is excluded from the 


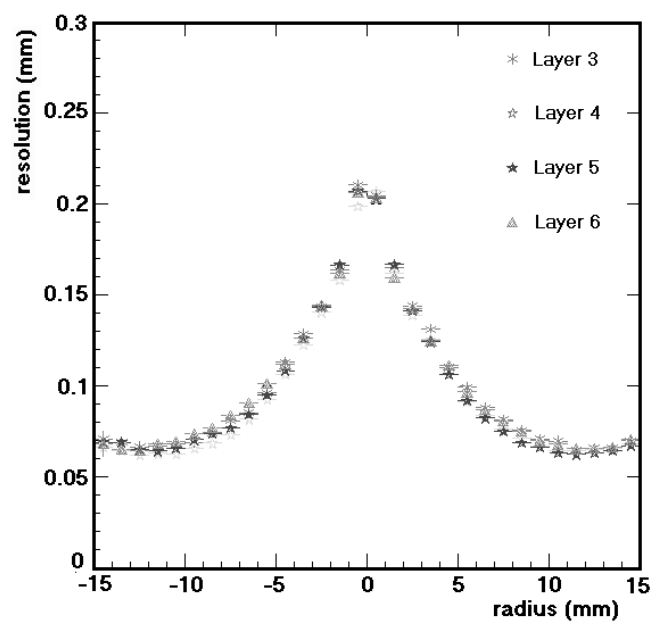

Fig. 9. The BIS-MDT Module-0 resolution as a function of the drift path for individual tubes belonging to four different layers.

fit and a straight line ${ }^{6}$ is computed from the remaining seven ones. This fitted line is compared with the excluded hit and the differences between the line and the measurement are plotted as a function of the drift path. These residuals are divided in slices of drift path, each corresponding distribution is fitted with a Gaussian, whose standard deviation represents the individual tube resolution for the given drift path, after subtracting in quadrature the contribution of the extrapolation error (Fig. 9). The resolution is computed separately for positive and negative residuals and the final result is given by the average value. The procedure is repeated to properly assign the measurement errors to the points used in the track fit.

The resolution deteriorates close to the wire, due to the lack of uniformity of the drift paths of the primary electrons and the charge fluctuations. Generally, the parameters that contribute to the spatial resolution are the gas gain fluctuations, the fluctuations of the electron cluster positions, the diffusion, the charge fluctuation, the electronic noise (tube resistor termination), and, -mainly for the low energy muons- the multiple scattering. As the signal is created after the arrival of the 20th electron, the first mentioned parameters make a dominant contribution to the resolution error near the wire. The multiple scattering effect dominates near to the tube wall, where the spatial resolution has smaller values and the contribution of this effect is comparable.

\section{F. Track Reconstruction}

The muon tracks are reconstructed using the correct $t_{0}$ values, the best $\mathrm{r}$-t relation and the MDT spatial resolution. In Fig. 10(a) and (b) some reconstructed tracks are shown. In Fig. 10(a) the track is not tangent to the isochronal curve and this is possibly because of the presence of a delta ray, which obscures the muon.

\section{G. Single Tube Efficiency}

A tube is considered inefficient when either no hit is present or the distance between the hit and the track is larger than three times the resolution at this distance.

${ }^{6}$ The $\chi^{2}$ (chisquare) fit that has been applied on the reconstructed tracks reduces the number of the accepted events by a factor, which approximately equals the number of low energy muons $(<1.6 \mathrm{GeV})$ as it comes out from the Monte Carlo simulation of the muon energy spectrum.

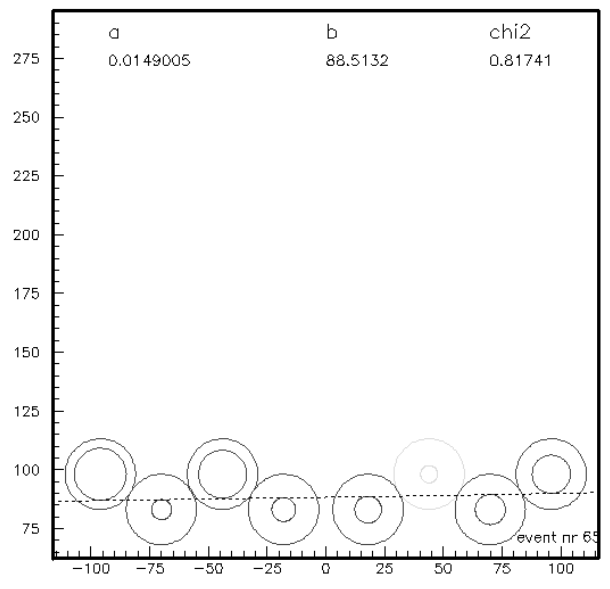

(a)

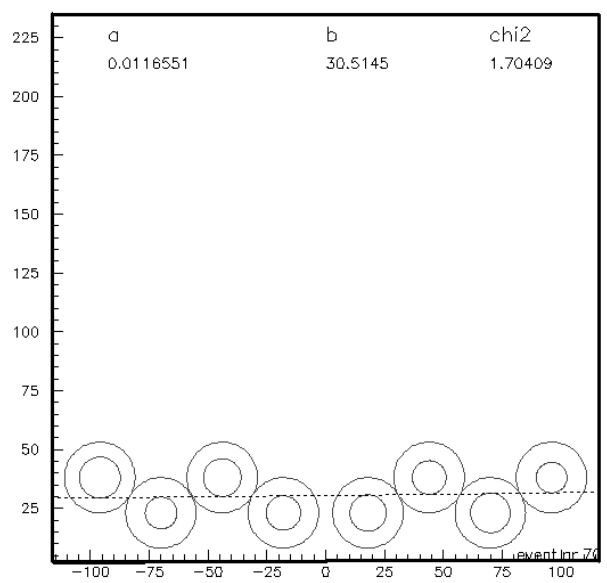

(b)

Fig. 10. Track reconstruction is shown in both (a)with seven drift tubes and in (b) with eight drift tubes.

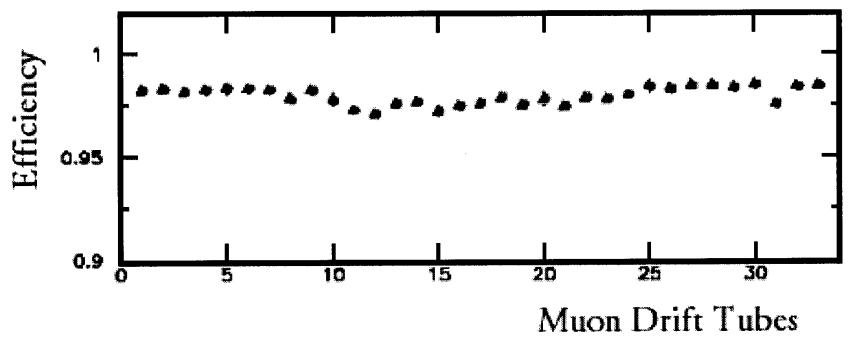

Fig. 11. Single tube efficiency, integrated over the full drift path, for a sample of 33 tubes of the BIS-MDT chamber.

As shown in Fig. 11, under this definition, the single tube efficiency for a typical sample of 33 tubes, almost equally distributed in eight layers, averaged over the full drift path is typically $97 \%-98 \%$.

Fig. 12 presents the efficiency as a function of the drift path for a single tube of the BIS-MDT chamber. The inefficiency close to the tube wall is clearly visible. The drop of the efficiency up to a $14 \mathrm{~mm}$ drift path is understood as due to $\delta$-ray production. After this distance the drop in the efficiency is due to the tube wall effect. 


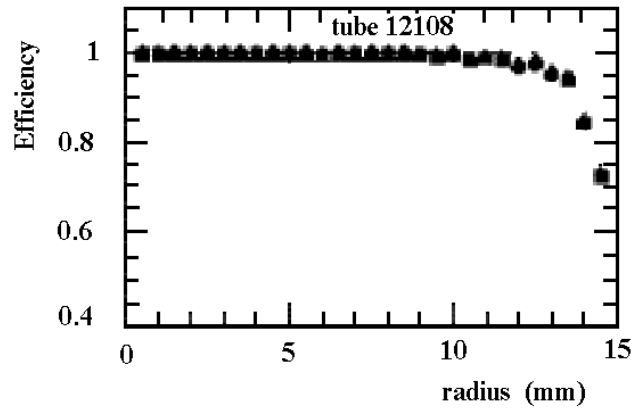

Fig. 12. Single tube efficiency as a function of the drift path.

\section{CONCLUSION}

The operation of one of the BIS-MDT chamber prototypes has been studied using cosmic rays. The variations of the time spectra have been studied using different discriminator thresholds and operation HV values. The tubes show, within errors, uniform performance concerning their drift time properties. As a consequence, the same $r$ - $t$ relation is obtained for all the tubes and after the autocalibration procedure is used for further analysis. Using the reconstructed tracks the chamber resolution is derived as a function of the drift path $(<100 \mu \mathrm{m}$ for $\mathrm{r}>5 \mathrm{~mm})$. The single tube efficiency is high $(\sim 98 \%)$ and deteriorates close to the walls. Overall, the cosmic ray study shows that the chamber Module-0 fulfills the ATLAS muon spectrometer requirements.

\section{ACKNOWLEDGMENT}

The authors would like to thank the technical staff-W. Andreazza, P. Kremizis, and K. Razakias, for their help on the installation and the setup. Special thanks to D. Pappas for his continuous help on the tube wiring and K. Ekonomou for his enormous help on the chamber construction and installation of the gas manifolds and the Faraday cage on the chamber. The on-chamber gas distribution was assembled, installed, and tested with the assistance of B. Pfeifer and J. Tobias, whom the authors cordially thank.

They would also like to thank A. Kojine and V. Goriatchev for providing the scintillators and helping in the first commissioning of the hodoscope, E. Christidi and S. Stefanidis for their contribution on the hodoscope setup, and J. Berbiers for his endless time of discussions and his technical assistance.

They are also grateful to the collaborators from Harvard, Boston, and Michigan Universities for providing the electronics and a lot of help during the debugging period. More specifically, they thank G. Novak for his valuable work on the electronics problems, without which the chamber would not have been operational.
Many thanks to R. Veenhof for his help on the "Garfield" simulation program.

Great assistance in understanding "Calib" $\mathrm{C}++$ code was provided by T. Baroncelli, M. Iodice, and D. Orestano. Many thanks to L. Pontecorvo and to many other collaborators for useful discussions.

\section{REFERENCES}

[1] “ATLAS, Muon Spectrometer, Tech. Design Rep.,", CERN/LHCC/ 97-22, ATLAS TDR 10, 1997.

[2] S. Agosteo et al., "A facility for the test of large area muon chambers at high rates: CERN-EP-2000-031, CERN-SL-2000-008-EA, Geneva, CERN, 16 Feb. 2000," Nucl. Instrum. Methods Phys. Res., vol. A: 452, no. 1-2, pp. 94-104, 2000.

[3] R. M. Avramidou, M. Dris, E. N. Gazis, O. Kortner, and S. Palestini, "Hodoscope Performance for the Cosmic Ray Setup of the MDT-BIS Chamber 'Beatrice',", ATL-COM-MUON-2003-002, 2002.

[4] F. Bauer et al., "The first precision drift tube chambers for the ATLAS muon spectrometer: ATL-MUON-2001-004, (MPI-PhE/2001-03), (ATL-COM-MUON-2001-015), 28 Feb. 2001,” Nucl. Instrum. Methods, vol. A478, pp. 153-157, 2000.

[5] D. Sampsonidis et al., "Construction of the BIS-MDT Chamber Module 0,", ATL-COM-MUON-2001-006, 2001.

[6] G. Kaptsis, C. Petridou, I. Tsiafis, and J. Wotschack, "Mechanical Design of the BIS-MDT Chamber Module Zero,", ATL-MUON-98-242, (ATL-COM-MUON-98-004), 1998.

[7] T. Alexopoulos et al., "The Quality Assurance_Quality Control of the Monitored Drift Tubes at the HEP Laboratory of the National Technical University of Athens,", ATL-MUON-2001-019, (ATL-COM-MUON2001-017), 2001.

[8] _ - "A quality assurance and quality control (QA QC) procedure of the monitored drift tubes (MDT) for the BIS-chambers of the ATLAS muon spectrometer," IEEE Trans. Nucl. Sci.-Nucl. Instrum. Methods, vol. 49, no. 5, pp. 2484-2487, Oct. 2002.

[9] R. Avramidou, E. N. Gazis, F. Rohrbach, S. Schuh, Y. Sedykh, and I. Vichou, "X-Ray Tomograph Study of the MDT-BIS Chambers 'Beatrice' and 'Camille',", ATL-COM-MUON-2003-001, 2002.

[10] K. Hagiwara, "Phys. Rev. D66 ," PDG, 2002.

[11] O. Kortner, MTGEANT-4, The Munich Test-Stand Simulation Programme: Manual version 1.0, 2000

[12] D. Fassouliotis, P. Ioannou, C. Kourkoumelis, V. Pancheluga, D. Pappas, S. Stefanidis, and V. Birioukov, "Improvements/Experience Derived from Wiring 10\% of BIS MDTs,", ATL-MUON-2001-005, (ATL-COM-MUON-2001-011), 2001.

[13] D. Fassouliotis, P. Ioannou, C. Kourkoumelis, and V. Birioukov, "MDT BIS Module 0 Tube Assembly,", ATL-MUON-2000-014, (ATL-COMMUON-99-031), 1999.

[14] Proc. 5th Workshop Electronics for LHC Experiments, Snowmass, CO, Oct. 1999, CERN 99-09, CERN/LHCC/99-33.

[15] Proc. 7th Workshop on Electronics for LHC Experiments, Stockholm, Sweden, Oct. 2001, CERN 2001-05, CERN/LHCC/21-34.

[16] Calib: A Package for MDT Calibration Studies-User Manual, 2002.

[17] R. Veenhof, "GARFIELD - Simulation of Gaseous Detectors,", CERN Program Library W5050, 2001.

[18] A. Biscossa et al., "Construction and test of a full scale prototype of an atlas muon spectrometer tracking chamber," Nucl. Instrum. Methods, vol. A425, pp. 140-164, 1999.

[19] — - "Calypso: A full scale MDT prototype for the ATLAS muon spectrometer," Nucl. Instrum. Methods, vol. A419, pp. 331-335, 1998.

[20] P. Creti, P. M. Cambiaghi, R. Ferrari, M. Fraternali, G. Gaudio, and A. Lanza, "Results from the 1998 Test Beam of the Calypso Chamber Filled With an $\mathrm{Ar}-\mathrm{CO}_{2}$ Gas Mixture,", ATL-MUON-2000-006, (ATL-COMMUON-99-033).

[21] F. Bauer et al., "Construction and test of MDT chambers for the ATLAS muon spectrometer," Nucl. Instrum. Methods Phys. Res., vol. A: 46, pp. $17-20,2000$. 